\title{
The terms of a language with one constant, one binary function, and one 4-ary function have an odd number of symbols
}

Open Mathematics Collaboration*†

September 5, 2021

\begin{abstract}
We show using induction on complexity that all terms of a language with one constant, one binary function, and one 4-ary function have an odd number of symbols.
\end{abstract}

keywords: language, induction on complexity, first-order logic

The most updated version of this white paper is available at https://osf.io/ue32a/download https: //zenodo.org/record/5457880

\section{Introduction}

1. This is a pedagogical white paper on first-order logic.

2. Our purpose is to discuss a result in [1] which is licensed under [2].

3. We use minimal notation but preserving all relevant mathematical information.

*All authors with their affiliations appear at the end of this white paper.

†Corresponding author: mplobo@uft.edu.br|Open Mathematics Collaboration 


\section{Meta-linguistic symbols}

4. $:=$ means that what is on the left is defined by what is on the right.

5. : झ means that the strings on both sides are identical.

6. $a, b \vdash c$ means deduction of $c$ from $a, b$.

\section{Proposition}

7.

$$
\mathscr{L}=\{0, f, g\} \rightarrow \text { every } \mathscr{L} \text {-term has an odd number of symbols }
$$

8. $\mathscr{L}:=$ language

9. $0:=$ constant symbol

10. $f:=$ binary function symbol

11. $g:=4$-ary function symbol

\section{Proof of (7) by induction on complexity}

12. $t:=\mathscr{L}$-term

13. We need to prove that $t$ has an odd number of symbols.

\section{Case 1}

14. $t: \equiv 0$

15. $t$ has 1 symbol.

\section{Case 2}

16. $t$ is a variable.

17. $t$ has 1 symbol. 


\section{Case 3}

18. $t: \equiv f t_{1} t_{2}$

19. $n:=$ number of symbols in $t$

20. Inductive hypothesis: a term has an odd number of symbols.

21. Let $n_{1}$ and $n_{2}$ be the number of symbols in $t_{1}$ and $t_{2}$, respectively.

22. (20) $\vdash n_{1}$ and $n_{2}$ are odd numbers.

23. $n=n_{1}+n_{2}+1$

24. (22), (23) $\vdash n$ is odd.

Case 4

25. $t: \equiv g t_{1} t_{2} t_{3} t_{4}$

26. This case is similar to Case 3 .

\section{Open Invitation}

Review, add content, and co-author this white paper [3,4]. Join the Open Mathematics Collaboration.

Send your contribution to mplobo@uft.edu.br.

\section{Open Science}

The latex file for this white paper together with other supplementary files are available in $[5,6]$. 


\section{How to cite this paper?}

https://doi.org/10.31219/osf.io/ue32a

https://zenodo.org/record/5457880

\section{Acknowledgements}

+ Center for Open Science https://cos.io

+ Open Science Framework https://osf .io

+ Zenodo https://zenodo.org

\section{Agreement}
All authors agree with [4].

\section{References}

[1] Leary, Christopher C., and Lars Kristiansen. A friendly introduction to mathematical logic, 2nd edition, 2015.

https://knightscholar .geneseo . edu/geneseo-authors/6

[2] CC. Creative Commons. https://creativecommons.org

[3] Lobo, Matheus P. "Microarticles." OSF Preprints, 28 Oct. 2019. https://doi.org/10.31219/osf.io/ejrct

[4] Lobo, Matheus P. "Simple Guidelines for Authors: Open Journal of Mathematics and Physics." OSF Preprints, 15 Nov. 2019. https://doi.org/10.31219/osf .io/fk836 
[5] Lobo, Matheus P. "Open Journal of Mathematics and Physics (OJMP)." OSF, 21 Apr. 2020. https://osf .io/6hzyp/files

[6] https://zenodo.org/record/5457880

\section{The Open Mathematics Collaboration}

Matheus Pereira Lobo (lead author, mplobo@uft.edu.br) $)^{1,2}$ https://orcid.org/0000-0003-4554-1372

${ }^{1}$ Federal University of Tocantins (Brazil)

${ }^{2}$ Universidade Aberta (UAb, Portugal) 